\title{
REST in Practice
}

\author{
Jim Webber \\ ThoughtWorks, 168-173 High Holborn, London, United Kingdom, WC1V 7AA \\ Jim.WebberaThoughtWorks.com
}

\begin{abstract}
The Web has emerged as a viable platform for building distributed systems beyond its traditional scope as a scalable means of sharing and disseminating information. In this paper I present observations from recent industrial development projects where commodity Web infrastructure and common patterns have been used to create large, scalable, and dependable computer systems.
\end{abstract}

Keywords: Dependability, ESB, REST, Scalability, SOA.

\section{Introduction}

Over the years we've seen many systems architecture approaches come and go as we've worked hard in IT to keep up with the pace of business change. While agile delivery methods have helped enormously on a project-by-project basis, enterprise system portfolios remain notoriously resistant to change.

Although we've seen significant progress in the way we analyze and govern enterprise systems through business-aligned SOA, the technology choices we make all too often undermine those efforts. But for almost two decades the enterprise architecture most of us have dreamed of has been sitting in plain sight. The Web has become the world's foremost example of a scalable, resilient, and loosely coupled system of systems, which are precisely the characteristics we want in enterprise solutions.

In contrast the Web succeeds by avoiding inappropriate technology choices, which bow to vendor pressure over business imperatives, while delivering all of the "ilities" that we demand from enterprise-grade systems. Not only is the Web more than a match for traditional middleware from a technology perspective, but from a cost perspective too. The keynote talk affiliated with this paper addresses financial and risk in software architecture using case studies from recent projects.

Acknowledgments. The author would like to thank Ali Babar and Ian Gorton for the opportunity to deliver these observations to ECSA and to the delivery teams at ThoughtWorks for sharing their delivery experiences. 